\title{
The Effect that the Application of Time-Based Electrolysis Has on Acute Ischemia
}

This neurological damage accelerates the infection reaction of cells and apoptosis at the time of reperfusion after ischemia occurs. BCL-2/BCL-2 allogeneic begeminum has a function of suppressing the apoptosis of cells, and thus it is inferred that the susceptibility of cells to apoptosis is determined by the amount of allogeneic begeminum present which is determined based on the amount of BAX. Ischemia was induced in SD mice by occluding the common carotid artery for 5 minutes, after which blood was re-perfused. NEES was applied to acupuncture points, at 12, 24, and 48 hours post-ischemia on the joksamri, Hapgok. Protein expression was investigated through BAX antibody immuno-reactive cells in the cerebral nerve cells and Western blotting.

The results were as follows:

In the present study as well, as a result of observation of the change in the number of the BAX reaction cells after the inducement of $\mathrm{Gl}$, there was the aspect of most of the BAX reaction cells being observed in the corpus striatum area of the Gl group 24 hours after the inducement of ischemia. This revealed the same results as those of previous studies in which the change in the number of BAX reaction cells occurred in all areas while ischemia was in progress. The change in the expression of BAX protein after 24 hours showed that there was a very significant reduction in the NEES group compared to the Gl group ( $p<.01$ ). As a result, a greatest amount of change in the number of BAX immunoreactive cells related to apoptosis 24 hours after ischemia appeared in the NEES group. This study that ischemia increases the expression of BAX that induces apoptosis. Thus, it is determined that ischemia is the main cause of the apoptosis of neurons, and this study reveals that low frequency needle electrode electrical stimulation has the effect of blocking the apoptosis of neurons by reducing protein related to the apoptosis of cells that has increased after ischemia has occurred.

Key words: Ischemia; Apoptosis; Needle Electrode Electrical Stimulation; Joksamri; Hapgok; BAX
Jung Sook Lee ${ }^{a}$, Young Wha Song $^{b}$, Sung Won Kim

${ }^{a}$ Dongju University College, Pusan; 'Dongnam

Health University, Suwon, Korea

Received : 8 June 2015

Revised : 11 July 2015

Accepted : 2 August 2015

Address for correspondence

Sung Won Kim, PT, Ph.D

Department of Physical Therapy,

Dongnam Health University, 50

Cheoncheon-ro 74-gil, Jangan-gu.

Suwon, Korea

Tel : 82-31-249-6447

Email : johnswkim@dongnam.ac.kr

\section{INTRODUCTION}

Ischemic Strokes are categorized as cerebral infarctions and temporary ischemic seizures. In the case that an ischemia of neurons occurs, the supply of oxygen to cells is blocked so that the ATP of microcondria becomes depleted, and acidosis occurs within cells caused by the increase in lactic acid due to anaerobic metabolism. When this condition is maintained for a long period, apopto- sis of cells occurs. In the case that ischemia and reperfusion occurs within a short period of time, the number of excitatory neurotransmitters of neurons increases. In addition, calcium flows into cells, which causes secondary neurological damage. It is believed that this neurological damage accelerates the infection reaction of cells and apoptosis at the time of reperfusion after ischemia occurs(1). BCL-2/BCL-2 allogeneic begeminum has a function of suppressing the apoptosis of cells, 
and thus it is inferred that the susceptibility of cells to apoptosis is determined by the amount of allogeneic begeminum present which is determined based on the amount of BAX(2). Thus, it follows that cells tend to experience apoptosis easily in the case that the amount of BAX relatively increases. And, cells tend to not experience apoptosis easily in the case that the amount of BCL-2 relatively increases. Apoptosis of cells is regulated by the protein BAX that boosts the selfdestruction of cells and the protein $\mathrm{Bd}-2$ that restrains the self destruction of cells. In addition, cytochrome $\mathrm{C}$ that has been removed activates caspase 9, which then activates caspase 3 and results in apoptosis of cells(3). BAX is an indicatorthat determines whether the neurons have been damaged (4). A recent report that states that the apoptosis of neurons due to natural causes was noticeably suppressed in test animals which had BAX genes removed clearly explains the effects of BAX (5).

BAX is one of the gene clusters of bcl-2 which synthesizes pro-apoptotic proteins, and the activity of the transcription of BAX as a transcriptional target is suppressed due to mutant p53. BAX maintains a parallel state by being combined with $\mathrm{Bcl}-\mathrm{XL}$, and when a large amount of BAX molecules are produced, the BAX molecules which have been removed become activated and activate a $\mathrm{P}$ number ICE/caspases which accelerates apoptosis of cells. Thus, this study researched the change of BAX that is related to the apoptosis of cells within the corpus striatum of the the common carotid artery of mice induced with ischemia of the low frequency needle electrode electrical stimulation (NEES) group and the ischemia inducement(GI) group. In addition, the differences at 12 hours, 24 hours and 48 hours after the inducement of ischemia and the effects of electrolysis after strokes were studied.

\section{METHODS}

\section{Subjects}

Subjects were 8 week old, specific pathogen free, male SD rats which had been allowed to adjust to the laboratory environment for over 1 week and weighed approximately $300 \mathrm{~g}$ (Orient BIO). The laboratory was kept at $22 \pm 1 \mathrm{C}$ and $45-55 \%$ humidity.

\section{Ischemia Induction Model and NEES}

Subjects were anesthetized using 3\% isoflurane (Choongwae Pharma Corportation, Korea). Incisions $2 \mathrm{~cm}$ in length were made in the center of both common carotid arteries, the vagus nerves were separated, and circulation in both arteries was completely cut off using non-absorbent thread. Five minutes later, the thread was removed to allow the reperfusion of blood.

The subjects were divided into the control group (no closing of the common carotid artery), the global ischemia (GI) group (common carotid artery closed), and the NEES group (NEES treatment applied after GI induction). Subjects in the GI group were allowed to recover for 30 minutes at 12,24 , and 48 hours after induction of GI with $3 \%$ isoflurane as an anesthetic. Subjects in the NEES group were given NEES treatment by using a needle electrode electrical stimulator (PG6, ITO, Japan, 9V) at the right and left Joksamri, Hapgok for 30 minutes in concurrence with anesthesia with $3 \%$ isoflurane at 12,24 , and 48 hours after induction of GI. NEES was done using a $2 \mathrm{~Hz}$ current, and strength was adjusted according to muscle movement around the stimulation point.

\section{Immunohistology}

Immunohistological studies were performed on striatum tissue samples of GI induced rats. Fixed brain tissue was freeze sectioned using 4\% paraformaldehyde, and the free floating method and a Vectastain ACB Kit (Vector, USA) was used. Anti-PARP was used as the primary anti body. After leaving the primaryanti body to react for 3 days at $4^{\circ} \mathrm{C}$, it was washed 3 times for 5 minutes each with 0.1M PBS, after which it was left to react with the secondary anti body for 1 day at 4 ${ }^{\circ} \mathrm{C}$. After DAB color development(Sigma,USA), tissue samples were put onto slides using $1 \%$ gelatin, dried in a dry oven for approximately 2 hours, dehydrated and madeclear, thenmounted using permount solution(Fisher, USA).

\section{Western Blotting}

The removed striatum was divided according to area and evenly broken down using a homogenizer. It was centrifuged at 1000 rpm and the supernatent was discarded, after which it was washed twice with cold PBS. After centrifuging, a Protein assay kit (Bio-rad) was used to measure the optical 
density of the protein at 750nm. The quantified protein was mixed with a lysis buffer and sample buffer (60mM tris; pH 6.8, $10 \%$ glycerol, $2 \%$ SDS, $0.01 \%$ bromophenol blue) in order to even the amount of protein, then boiled at $100 \mathrm{C}$ for 5 minutes on a heat block. This was centrifuged and the specimein collected. A separating gel (12-15\%) was made by pouring into a clean electrophoresis glass plate a combination of $30 \%$ polyacrylamide mix, thrice distilled water, $1.5 \mathrm{M}$ tris $(\mathrm{pH} 8.8), 10 \%$ SDS, 10\% ammonium persulfate (produced that day), and TEMED. $10 \mathrm{x}$ stock of electrophoresis running buffer was made by melting $30.0 \mathrm{~g}$ tris base, $144 \mathrm{~g}$ glycine, and 10g SDS into $1 \mathrm{~L} .10-20 \mu \mathrm{L}$ of the quantified protein was added, and $100 \mathrm{~V}$ was run for approximately 1 hour. The transfer buffer was made by adding $3.03 \mathrm{~g}$ tris base, $14.63 \mathrm{~g}$ glycine, and $200 \mathrm{~mL}$ methane to $1 \mathrm{~L}$. It was stored at $4 \mathrm{C}$ before use. The thin membrane was washed with a TTBS solution ( $\mathrm{pH}$ 7.5) composed of $200 \mathrm{mM}$ tris-base, $1.54 \mathrm{M} \mathrm{NaCl}$, thrice distilled water, and 20 tween. It was them put into $5 \%$ lac defloratum and left for a day at $4 \mathrm{C}$.

The next day, after removing the blocking solution, a primary anti body identical to the one used in immune staining was reacted for 1 hour with the thin membrane put into a solution made by diluting 5\% lac defloratum to 1000 times its original concentration. This was then washed 3 times with TTBS solution for 10 minutes each. A secondary anti body was reacted for 1 hour with the thin membrane put into a solution made by diluting 5\% lac defloratum to 1000 times its original concentration. After removing the solution, this was then washed 3 times with TTBS solution for 10 minutes each. Solutions A and B from the ECL kit were well combined at a ratio of $40: 1$ and this was used to wet the thin membrane. After 1 hour, the thin membrane was put on a cassette and photo sensitized using $x$ ray film. After photosensitizing for a period of time, expression bands were checked and a fixator was used for fixing. After fixing, the bands were washed in clean running water, dried, scanned, and a concentration meter (Bio-rad) was used to measure the optical density of each band.

\section{Data Analysis}

All data collected in this study were encoded and analyzed using a computerized statistical analysis program (SPSS 12.0K/PC). In order to find out the effects of the control group, global ischemia group, and NEES group, the level of significance was set to $\alpha=.05$. ANOVA was used to analyze the differences between the three groups.

\section{RESULTS}

\section{The Change in the Number of the BAX Reaction Cells of Corpus Striatum}

After the inducement of ischemia, the number of the BAX reaction cells of the corpus striatum of mice as can be seen in Fig. 1 was $67.67 \pm 3.05$ in the control group. 12 hours after the inducement of ischemia, the number of the BAX reaction cells in the GI group and the NEES group was 109.67 \pm 7.57 and94.67 \pm 3.51 respectively, which showed a reduction in the NEES group compared to the GI group. The number of the BAX reaction cells 24 hours after the inducement of ischemia in the GI group and NEES group was 136.67 \pm 10.60 and106.00 \pm 3.60 respectively, which showed a significant reduction $(p<.05)$ in the NEES group compared to the GI group. 48 hours after the inducement of ischemia, the number of the BAX reaction cells was $97.67 \pm 6.11$ and79.67 \pm 7.50 in the GI group and NEES group respectively, which showed a significant reduction $(\mathrm{p}<.05)$ in the NEES group compared to the GI group. In addition, the number of BAX reaction cells 24 hours after the inducement of ischemia was $136.67 \pm 10.60$ and104.67 \pm 5.50 in the GI group and NEES group respectively, which showed a significant reduction $(p<.05)$ in the NEES group compared to the GI group. However, in comparison of spots on the body related to acupuncture, the number of BAX reaction cells 24 hours after the inducement of ischemia was 106.00 \pm 3.60 in the NEES group and $104.67 \pm 5.50$ in the NEES group, in which there was not a significant difference between Joksamri and Hapgok. 


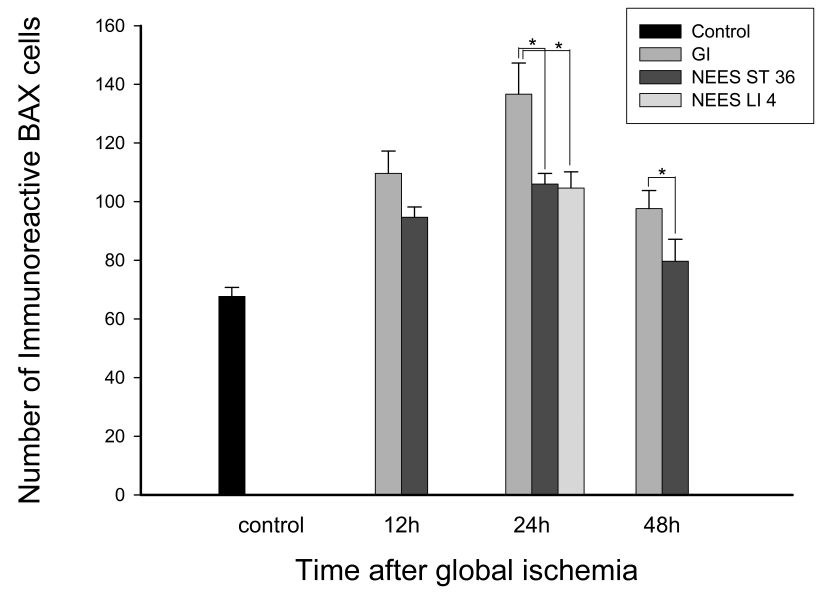

Fig. 1. Effect of needle electrode electrical stimulation on BAX immunoreactive cells in rat striatum after transient global ischemia. Control=no surgical procedure; Gl=transient global ischemia; NEES ST $36=$ needle electrode electrical stimulation at joksamri, NEES LI 4 = needle electrode electrical stimulation at Hapgok. Values are the mean \pm SE. *p<.05.

\section{Change in the Expression of BAX Protein of the Corpus Striatum}

After the inducement of ischemia, the expression of the BAX reaction cells of the corpus striatum of mice as can be seen in Fig. 2 was $44.35 \pm 3.11$ in the control group. 12 hours after the inducement of ischemia, the expression of the BAX reaction cells in the GI group and the NEES group was $72.06 \pm 3.45$ and $67.99 \pm 4.32$ respectively, which showed a reduction in the NEES group compared to the GI group. The expression of the BAX reaction cells 24 hours after the inducement of ischemia in the GI group and NEES group was $84.34 \pm 3.65$ and $56.21 \pm 5.65$ respectively, which showed a very significant reduction $(p<.01)$ in the NEES group compared to the GI group. 48 hours after the inducement of ischemia, the expression of the BAX reaction cells was $71.87 \pm 3.79$ and61.32 \pm 2.49 in the GI group and NEES group respectively, which showed a very significant reduction $(p<.01)$ in the NEES group compared to the GI group.

In addition, the expression of BAX reaction cells 24 hours after the inducement of ischemia was $84.34 \pm 3.65$ and $76.24 \pm 3.88$ in the GI group and the NEES group respectively, in which there was not a significant difference between the two groups. In comparison of spots on the body relat- ed to acupuncture, the expression of BAX reaction cells 24 hours after the inducement of ischemia

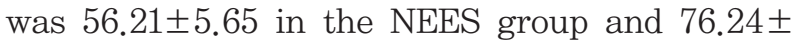
3.88in the NEES group, in which there was not a significant difference between Joksamri and Hapgok.

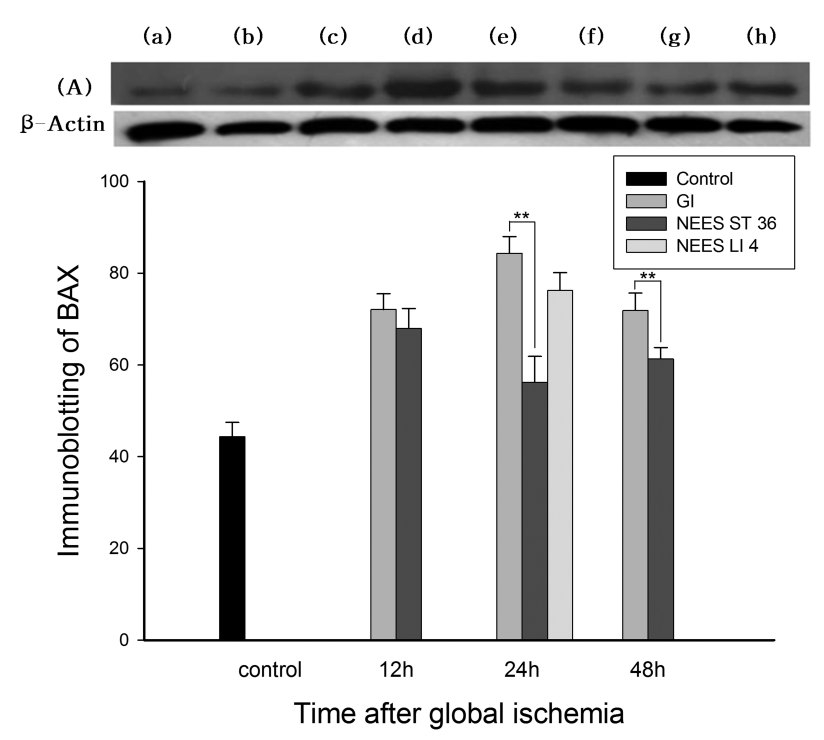

Fig. 2 Changes of BAX expression in rats striatum after transient global ischemia.

(A) Total BAX protein was extracted from striatum.

(a) Control group,

(b) $12 \mathrm{~h}$ after transient global ischemia,

(c) needle electrode electrical stimulation (Joksamri) at $12 \mathrm{~h}$ after transient global ischemia,

(d) $24 \mathrm{~h}$ after transient global ischemia,

(e) needle electrode electrical stimulation (Joksamri) at $24 \mathrm{~h}$ after transient global ischemia,

(f) needle electrode electrical stimulation (Hapgok) at $24 \mathrm{~h}$ after transient global ischemia,

(g) $48 \mathrm{~h}$ after transient global ischemia,

(h) needle electrode electrical stimulation (Joksamri) at $48 \mathrm{~h}$ after transient global ischemia.

(B) Results are expressed as density. Control=no surgical procedure; Gl=transient global ischemia; NEES ST 36 = needle electrode electrical stimulation at joksamri, NEES LI 4 = needle electrode electrical stimulation at Hapgok. ${ }^{* *}$ p<.01, ${ }^{*}$ p<.05. 


\section{DISCUSSION}

Stress in the form of neurotransmitters of brain cells, depolarization, the inflow of calcium and the like cause a biochemical change within cells, and genes which quickly respond to biochemical change are called immediate early genes(IEGs)(6).

BAX is a protein that has similarities with Bcl-2 and forms a heterodimer together with a homodimer or Bcl-2. The expression of BAX increased in the cerebral infarction area at the time of cerebral ischemia in white mice(7), and the expression of the BAX protein of corpus striatum and the number of cells that reacted to Anti-BAX after the inducement of forebrain ischemia increased (Fig. 1, 2).

IEG p53 regulates the ratio of $\mathrm{Bcl}-2 / \mathrm{BAX}$, and the increase in the proportion of BAX induces apoptosis of cells(8). BAX that is excessively expressed in a diseased state suppresses the cell protection activity of Bcl-2 and thus induces apoptosis of cells(9). Proteins in the Bcl-2 family release cytochrome $\mathrm{c}$ from the mitochondria into the cytoplasm, and they regulate apoptosis through the promotion of BAX and the suppression of $\mathrm{Bcl}-\mathrm{xl}(10,11)$. Upon the release of cytochrome c into the cytoplasm, caspase that induces apoptosis is activated and apoptosis occurs.

It has been reported that in test animals in which BAX genes were removed, the apoptosis of neurons was noticeably suppressed(12). In addition, upon blocking the middle cerebral artery of white mice, low frequency needle electrode electrical stimulation delayed the expression of BAX (13). In terms of forebrain ischemia, it was shown that there was a neuroprotective effect when low temperature reduced the expression of the BAX protein(14). In the present study as well, as a result of observation of the change in the number of the BAX reaction cells after the inducement of GI, there was the aspect of most of the BAX reaction cells being observed in the corpus striatum area of the GI group 24 hours after the inducement of ischemia. This revealed the same results as those of previous studies in which the change in the number of BAX reaction cells occurred in all areas while ischemia was in progress. In addition, in regards to the effectiveness of NEES, it was shown that the number of BAX reaction cells in the corpus striatum area 24 hours after the inducement of ischemia was significantly reduced in both Joksamri and Hapgok of the NEES group in comparison to the GI group. The change in the expression of BAX protein after 24 hours showed that there was a very significant reduction in the NEES group compared to the GI group(p<.01). As a result, a greatest amount of change in the number of BAX immunoreactive cells related to apoptosis 24 hours after ischemia appeared in the NEES group.

As mentioned above, it has been shown in this study that ischemia increases the expression of BAX that induces apoptosis. Thus, it is determined that ischemia is the main cause of the apoptosis of neurons, and this study reveals that low frequency needle electrode electrical stimulation has the effect of blocking the apoptosis of neurons by reducing protein related to the apoptosis of cells that has increased after ischemia has occurred.

\section{CONCLUSION}

In the present study as well, as a result of observation of the change in the number of the BAX reaction cells after the inducement of GI, there was the aspect of most of the BAX reaction cells being observed in the corpus striatum area of the GI group 24 hours after the inducement of ischemia. This revealed the same results as those of previous studies in which the change in the number of BAX reaction cells occurred in all areas while ischemia was in progress. The change in the expression of BAX protein after 24 hours showed that there was a very significant reduction in the NEES group compared to the GI group(p<.01). As a result, a greatest amount of change in the number of BAX immunoreactive cells related to apoptosis 24 hours after ischemia appeared in the NEES group. This study that ischemia increases the expression of BAX that induces apoptosis. Thus, it is determined that ischemia is the main cause of the apoptosis of neurons, and this study reveals that low frequency needle electrode electrical stimulation has the effect of blocking the apoptosis of neurons by reducing protein related to the apoptosis of cells that has increased after ischemia has occurred. 


\section{REFERENCES}

1. Du C, Hu R, Csernansky CA, Hsu CY and Choi DW. Very delayed infarction after mild focal cerebral ischemia a role for apoptosis. J Cereb Blood Flow Metab 1996; 16: 195 - 201.

2. Oltvai ZN, Milliman CL, Korsmeyer SJ. BCL-2 heterodimerizes in vivo with a conserved homolog, BAX, that accelerates programmed cell death. Cell 1993; 74: 609-619.

3. Zheng Z, Zhao H, Steinberg GK, Yenari MA. Cellular and molecular events underlying ischemia-induced neuronal apoptosis. Drug News Perspect 2003; 16: 497-503.

4. Hara AT, Iwai and Niwa M. Immunohistoc hemical detection of BAX and Bcl-2 proteins in gerbil hppocampus followiong transient forebrain ischemia. Brain Res 1996; 711: 249-253.

5. Gibson ME, Han BH, Choi J, Knudson CM, Korsmeyer SJ, Parsadanian M, Holtzman DM. BAX contributes to apoptotic-like death following meonatal hypoxia-ischemia evidence for distinct apoptosis pathways. Mol Med 2001; 7(9): 644-655.

6. Herdegen T, Kovary, Buhl A, Basal expressionof the inducible transcription factors c-Jun, Jun-B, Jun D, c-Fos, Fos B and Knox-24 in the adult rat brain. J Comp Neurol 1995; 354: 39-59.

7. Gillardon F, Lenz C, Waschke KF, Krajewski S, Reed JC, Zimmermann M, Kuschinsky W. Altered expression of Bcl-2, Bcl-X, Bax, and c-Fos colocalizes with DNA fragmentation and ischemic cell damage following middle cerebral artery occlusion in rats. Mol Brain Res 1996; 40: $254-260$.
8. Chae $\mathrm{CH}$, The effect of DL-a-lipoic acid supplementation and exercise on bax, bcl-2 mRNA expression and caspase -3 activity in D-galactose induced aging. Graduate school of Sung Kyun Kwan University 2005.

9. Oltvai ZN, Milliman CL, Korsmeyer SJ, Bcl-2 heterodimerizes in vivo with a conserved homolog bax that accelerates programmed cell death. Cell 1993; 74: 609-619.

10. Kluck RM, Bossy-Wetzel E, Green DR. The release of cytochrome c from mitochondria; a primary site for Bcl-2 regulation of apoposis. Science 1997; 275: 1132-1136.

11. Kharbanda S, Pandey P, Sshofield L. Role for $\mathrm{Bcl}-\mathrm{xL}$ as an inhibitor of cytosolic cytochrome C accumulation in DNA damageinduced apoptosism. Proc Natl Acad Sci USA 1997; 94: 69396942.

12. Gibson ME, Han BH, Choi J, Knudson CM, Korsmeyer SJ, Parasdanian M, Hotzman DM. Bax contributes to apoptotic-like death following neonatal hypoxia-ischemia, evidence for distinct apoptosis pathways. Mol Med 2001; 7(9): 644655.

13. Kim SW. An Effect of Needle Electrode Electrical Stimulation on Inhibition of Neuronal Cell death after Inducing Global Ischemia in the Rat Brain. Graduate school of Dong Eui University 2008.

14. Lee YS, Park SH, Yun BW, Noh JK. The Effect of Duration of Ischemia and Body Temperature on

19. the Expression of Bax/Bcl-2 in Transient Global Ischemia. Journal of Clinical Neurology 2000; 18(4): 431-438. 